\title{
ARTICLE
}

\section{Presence of Parent, Gender and Emotional Valence Influences Preschoolers' PFC Processing of Video Stimuli}

Justin R. Durnford ${ }^{\mathrm{a}}$,Jan Paolo M. Balagtas ${ }^{\mathrm{a}}$, Atiqah Azhari ${ }^{\mathrm{a}}$, Mengyu Lim ${ }^{\mathrm{a}}$,Giulio Gabrieli $^{\mathrm{a}}$, Andrea Bizzego ${ }^{\mathrm{b}}$ and Gianluca Esposito ${ }^{\mathrm{a}}$, b, c

${ }^{a}$ Psychology Program, School of Social Sciences, Nanyang Technological University, Singapore, Singapore; ${ }^{b}$ Department of Developmental and Social Psychology, University of Trento, Rovereto TN, Italy; ' Lee Kong Chian School of Medicine, Nanyang Technological University, Singapore, Singapore

\section{ARTICLE HISTORY}

Accepted August 9, 2020

Journal: Early Child Development and Care, Taylor \& Francis

\begin{abstract}
Due to an unprecedented increase in children's exposure to a wide range of video stimuli, there is a need to understand how they process them. From the tender age of 3 , children show distinct activations in the prefrontal cortex (PFC) when presented with children's movies. However, there are multiple factors that can influence child neural response to such screen media, namely, presence of a specific parent, gender differences and emotional valence. Sixty-two preschool children (37 boys) between ages 3 and 4 inclusive and their parents (33 mothers, 29 fathers) were recruited to engage in a joint video attention task involving three 1-min animation video clips that varied in emotional valence (positive, neutral, negative) while the children's neural responses were measured using functional near-infrared spectroscopy (fNIRS). We found a significant interaction effect between emotional valence and gender. Specifically, girls showed significantly more whole-PFC activity than boys when viewing the video clip with positive emotional valence. Children who engaged in joint-viewing with their fathers also showed significantly stronger PFC activity than with their mothers, regardless of the emotional valence of video. Our findings suggest how, at a PFC level, different factors interact with one another and influence the joint-viewing experience of screen time amongst mother-child and father-child dyads. The educational and applied implications of our findings will be discussed below.
\end{abstract}

\section{KEYWORDS}

Prefrontal Cortex; Preschool; fNIRS; Videos; Gender; Parent

\section{Introduction}

In modern times, children are being exposed to an unprecedented amount of diverse, multimodal screen media, such as films and television programmes (Carter, Rees, Hale, Bhattacharjee, \& Paradkar, 2016). Such stimuli elicit stronger emotional reactions than what static facial expressions alone are capable of (Schaefer, Nils, Sanchez, \& Philippot, 2010). Unfortunately, there is still a lack of studies examining how preschool children respond to such stimuli, especially on a neural level. More research in this 
area can give us a clear and realistic picture of the dynamic approach the human brain adopts in analysing information (Hasson \& Honey, 2012).

The Prefrontal Cortex (PFC). The preschool age is when the PFC experiences tremendous growth, as seen in better performance in neuropsychological tasks pertaining to PFC function (Luciana \& Nelson, 1998), anatomical studies (Gogtay et al., 2004), and neuroimaging evidence that points to improved functioning of the inferior prefrontal regions during the preschool years (Moriguchi \& Hiraki, 2009). Emotional content is commonly found in films and television (TV) programmes, and being capable of recognising emotional cues and inferring the characters' mental states requires the development of the Theory of Mind (TOM), which is the ability to assign mental states to oneself and others (Premack \& Woodruff, 1978). It is dependent on a network of brain regions that include the medial PFC, temporo-parietal junction (TPJ) and the precuneus (Saxe \& Kanwisher, 2003; Yeshurun, Nguyen, \& Hasson, 2017). A study conducted by Richardson and colleagues discovered that 3-year olds do have a significantly developed TOM that aids them in being cognizant of the character's emotions in films (Richardson, Lisandrelli, Riobueno-Naylor, \& Saxe, 2018). While viewing emotional content, preschoolers perform a substantial amount of emotional regulation, which overlaps with self-regulation (McClelland et al., 2018). Given that the PFC is responsible for the development of executive function (Baptista et al., 2017) and thus a preschooler's ability to conduct self-regulation (Blair, 2016), we can see that there is an important link between the development of the PFC, executive function and self-regulation in the preschool years (McClelland et al., 2018). Therefore, our study aims to have a closer look at how preschool children process video stimuli at a PFC level. In this field of research, there are three factors that have not been thoroughly studied yet.

\subsection{Factors Influencing PFC Processing of Videos}

The Presence of a Specific Parent. There is a disparity in the amount of research on how fathers can impact their children's emotional processing, as most research has only focused on mothers (Meuwissen, 2017). With a growing emphasis on father-child attachment (Cabrera, Tamis-LeMonda, Bradley, Hofferth, \& Lamb, 2000), it would be important for us to look at possible parental differences in this aspect. After all, as preschool children have a less developed prefrontal cortex to facilitate regulation of their own emotions (Gogtay et al., 2004), their parent's presence can be a form of social regulation that aids them in processing emotions (Reeck, Ames, \& Ochsner, 2016). However, it appears that mothers influence their child's emotion processing more than fathers do. Children have been found to be more upset by their mother's indifference (Kiel \& Kalomiris, 2015), and their executive functioning and thus emotional processing are only significantly affected by their mother's use of mental-state talk (Baptista et al., 2017). Such evidence depicts children's greater reliance on their mothers for optimal emotional processing. Consequently, as emotional-regulation following exposure to emotional stimuli can be detected as PFC activity (Ochsner \& Gross, 2005), the preschoolers' PFC would be stimulated more, so as to compensate for lower levels of social regulation from their fathers (Coan, 2008).

Gender Difference. While many studies did not use video stimuli, they do give us a clue on a possible gender difference. A meta-analysis has shown that from infancy to adolescence, girls have a small yet significant edge over boys at facial expression processing (FEP) (McClure, 2000). Research has shown that preschool girls have a slight 
edge over preschool boys in emotion understanding and false belief tasks (Charman, Ruffman, \& Clements, 2002; Walker, 2005). Therefore, it seems that preschool girls could be more efficient in emotional processing. Separately, studies have shown that as children develop from infancy through school age, they have a tendency to be better at processing words and faces that are positively valenced (Gao, Maurer, \& Nishimura, 2010; Kauschke, Bahn, Vesker, \& Schwarzer, 2019; Lepp anen \& Hietanen, 2004). This would mean that stimuli with a positive emotional valence would be processed better than others. Therefore, we should only see a gender difference in the PFC levels where video stimuli with positive emotional valence are concerned.

Emotional Valence. Emotional valence is the intrinsic affective quality of a stimulus (Frijda et al., 1986), making it appear positive (e.g. portraying happiness, etc.), neutral, or negative (e.g. portraying anger, sadness, etc.). Where children are concerned, their ability to process emotions embedded in stimuli will determine how well they can understand emotional cues. This shapes their emotional competence, thereby possibly influencing how they empathise with others and perceive their emotions correctly according to a given context (Cherland, 2004). That being said, it is proven that the PFC plays a role in processing the emotional valence of stimuli. A study discovered that the lateral PFC is more strongly activated when viewing negatively valenced pictures, while the medial PFC is recruited more when viewing positively valenced pictures (Kensinger \& Schacter, 2006). As such, the preschool children's PFC levels could vary when viewing videos with different emotional valences.

\subsection{Specific Aims and Hypotheses}

Therefore, for our study, we would expect preschool children to demonstrate higher PFC levels when they engage in joint viewing of the stimuli with their fathers as opposed to their mothers. As for our second hypothesis, our first hypothesis states that we would expect preschool girls to show higher PFC levels than preschool boys, only when viewing stimuli with positive emotional valence. Lastly, we suspect that there will be systematic differences in PFC levels across each level of emotional valence.

\section{Materials and Methods}

\subsection{Participants}

Sixty-two parent-child dyads comprising 29 father-child dyads (11 girls, 18 boys) (see Table 1) and 33 mother-child dyads (14 girls, 19 boys) (see Table 2) were recruited through online forums and social media platforms. To meet the requirements of our study, all participants had to be residing in Singapore, and parents had to be above 21 years of age, with their biological child aged between 36 to 48 months upon testing. All participants were screened for cognitive deficits, visual or hearing impairments, or major diseases that could hinder their performance in the experiment. We obtained informed consent from all our participants and remunerated them at the end of the study. All methods were performed in accordance with the relevant guidelines and regulations as well as following the Helsinki declaration. The study was approved by the Institutional Review Board of Nanyang Technological University (IRB-2018-06016). All data are available at this URL: https://doi.org/10.21979/N9/CTR0YX and https://doi.org/10.21979/N9/PFHB88. 
Table 1. Sociodemographic information of participants from the fatherchild sample $(\mathrm{N}=29)$

\begin{tabular}{llc}
\hline Age (Father) & $38.1 \pm 3.67$ years & \\
Age (Child) & $42.2 \pm 5.25$ months & 8 \\
\hline \multirow{3}{*}{ Educational Level } & Graduate/Professional degrees & 12 \\
& Standard College (BA/BSc.) & 2 \\
& Partial College (at least one year) & 7 \\
\hline & Secondary/ITE/Polytechnic/Pre-University & 26 \\
Employment Status & Employed Full-time & 1 \\
& Employed Part-time & 2 \\
\hline & Self-employed & 9 \\
& $\$ 1,000-\$ 4,999$ & 8 \\
& $\$ 5,000-\$ 8,999$ & 5 \\
Household Income & $\$ 9,000-\$ 12,999$ & 3 \\
& $\$ 13,000-\$ 19,999$ & 3 \\
\hline
\end{tabular}

Table 2. Sociodemographic information of participants from the motherchild sample $(\mathrm{N}=33)$

\begin{tabular}{llc}
\hline Age (Mother) & $34.8 \pm 4.22$ years & \\
Age (Child) & $42 \pm 6.09$ months & 9 \\
\hline \multirow{3}{*}{ Educational Level } & Graduate/Professional degrees & 17 \\
& Standard College (BA/BSc.) & 7 \\
\hline \multirow{3}{*}{ Employment Status } & Secondary/ITE/Polytechnic/Pre-University & 18 \\
& Employed Full-time & 2 \\
& Self-employed & 7 \\
& Unemployed/Homemaker & 6 \\
\hline \multirow{3}{*}{ Household Income } & $\$ 1,000-\$ 4,999$ & 5 \\
& $\$ 5,000-\$ 8,999$ & 12 \\
& $\$ 9,000-\$ 12,999$ & 11 \\
& $\$ 13,000-\$ 19,999$ & 2 \\
& $\$ 20,000$ & 2 \\
\hline
\end{tabular}




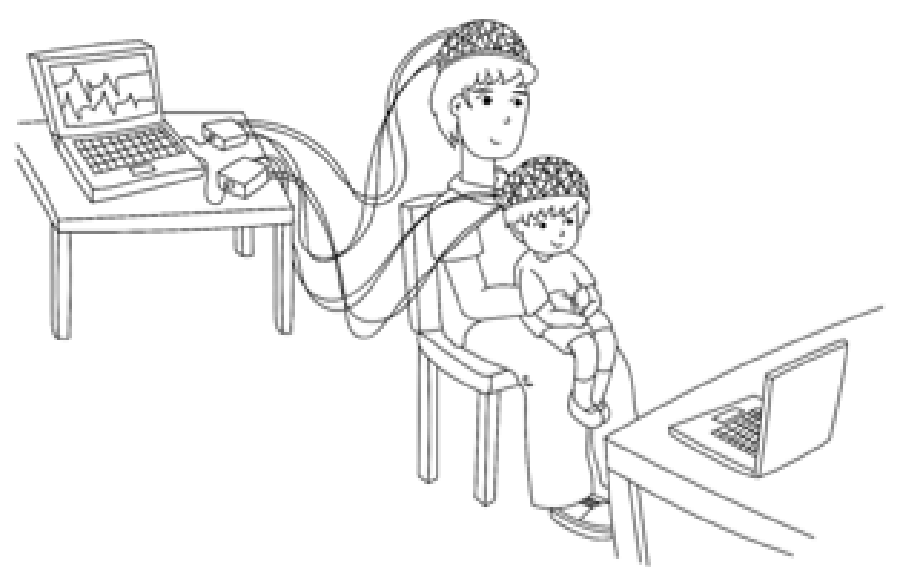

Figure 1. Experimental set-up and seating arrangement of father-child dyad. Figure illustrated by Farouq Azizan.

\subsection{Experimental Procedure}

Parents completed a demographic questionnaire online. Next, the dyads were invited to a child-friendly laboratory where they were greeted by a research assistant who briefly described the experiment and obtained informed consent before leading them into the experimental room. Afterwards, parents were asked to sit on a chair, with their child sitting on their lap throughout the experiment (see Figure 1). After which, the NIRS caps were fitted onto their heads, with recording conducted in tandem hyperscanning mode. A short video clip from the movie Moanna was screened to keep the dyads occupied so that the researchers could set up the NIRS devices and adjust the optodes accordingly. The videos were shown via a 15-inch Acer nitro laptop that was positioned on a table at about $40 \mathrm{~cm}$ from where the dyad sat. This same laptop was used to display our video sequences. Upon starting the fNIRS data-recording and the video sequences, all researchers exited the experimental room and re-entered only at the end of the video sequences. After which, the NIRS caps were removed, and the parents were debriefed and remunerated.

\subsection{Video Stimulus}

Dyads were engaged in a joint video attention task involving three 1-min animation video clips, one each from Brave, Peppa Pig and The Incredibles (see Table 3). These are well-known children animation movies and television programmes, and the selected video clips have been validated for depicting different emotional contexts (e.g. happy, neutral, angry)(Azhari et al., 2019), thereby increasing the generalisability of the recorded PFC levels beyond the laboratory setting. The clip from Brave had the most amount of positive expressions, consisting of smiles, hugs and laughter, and was considered a positive stimulus. The clip from Peppa Pig depicted a didactic family scene and consisted of a moderate amount of positive expressions, and was therefore labelled as neutral. Finally, the clip from The Incredibles depicted a family argument and had the least amount of positive expressions, and was considered a negative angry stimulus. The video stimulus presentation was preceded by a 5 -s fixation cross, and a 10-s inter-stimulus (ISI) fixation cross was added between each clip (see Figure 2) (Azhari et al., 2019). In order to minimise order effects, the sequence of the animation 
Table 3. Summary table of properties of video stimuli.

\begin{tabular}{lccc}
\hline Video Stimulus & $\begin{array}{c}\text { Video Complexity }^{a} \\
\text { (bytes) }\end{array}$ & $\begin{array}{c}\text { Audio Fundamentals } \\
(\mathrm{Hz})\end{array}$ & $\begin{array}{c}\text { Audio Intensity }^{b} \\
\left(\mathrm{~W} / \mathrm{m}^{2}\right)\end{array}$ \\
\hline Brave & 658119.31 & 245.26 & 59.89 \\
Peppa Pig & 468369.81 & 218.37 & 62.50 \\
The Incredibles & 423005.66 & 271.64 & 56.30 \\
\hline
\end{tabular}

${ }^{a}$ Bytes is a unit of memory size

${ }^{b} \mathrm{~W} / \mathrm{m}^{2}$ is a measure of sound energy

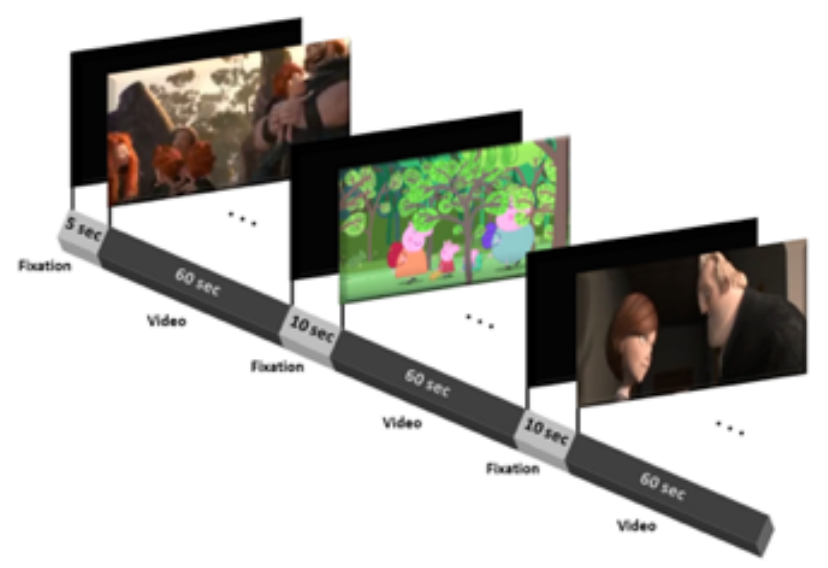

Figure 2. Video stimuli presentation. Father-child dyads were randomly assigned to one of six video sequences. From (Azhari et al., 2019).

clips was counterbalanced creating a total of six possible permutations (i.e. happyangry-neutral, angry-neutral-happy, etc.). Dyads are then randomly assigned to view one of those six video sequences. All video clips were screened on the same 15-inch Acer nitro laptop, with the brightness and volume set at 165 nits and $44 \mathrm{~dB}$ respectively.

Two properties of the stimuli, video and audio complexity, were quantified. Visual complexity measures the extent of visual cluttering and areas of higher information density within the scene (Bucy, Lang, Potter, \& Grabe, 1999). Audio complexity is defined as the root mean square (RMS) amplitude of the sound signal and reflects differences in acoustic energy (Denison, Driver, \& Ruff, 2013). Visual complexity of the video clips was calculated with Python and FFmpeg (v. 3.4.4), while audio complexity was calculated on Praat (v. 6.0.46) after prior conversion of the video clips to an audio format on FFmpeg. Visual and audio complexities are reported in Table 3 and were included as controls.

Where emotional valence is concerned, anger, fear and sadness are typically categorised as negatively valenced, whereas joy and pleasure are regarded as positively valenced emotions (Frijda et al., 1986). Two independent coders determined the emotional valence of all three videos through second-by-second coding of the video clip, where they indicated whether each second of the clip was positively valenced. The sum of the second-by-second coding across the entire clip was then used as a measure of positive valence. Inter-rater agreement between the two coders was calculated using 
the "irr" package in RStudio (Gamer, Lemon, Gamer, Robinson, \& Kendall's, 2012). An inter-rater agreement of at least $80 \%$ was achieved across the three videos.

\subsection{Functional Near-Infrared Spectroscopy (fNIRS)}

fNIRS recording was conducted in tandem hyperscanning mode at a scan rate of $7.81 \mathrm{~Hz}$ (NIRSport, NIRx Medical Technologies LLC). 8 LED sources (wavelengths of $760 \mathrm{~nm}$ and $850 \mathrm{~nm}$ ) and 7 detectors were arranged on the caps according to a standard PFC montage to form 20 channels on each cap (NIRS v.205 software). Distances between source and detector pairs were under an optimal maximum of $3 \mathrm{~cm}$.

fNIRS pre-processing was done on the NIRSLab software (NIRS v.205 software) (Azhari et al., 2020). Markers that denote the onset of each video stimulus were added to the time-series signals. Upon visual inspection of the signal, discontinuities and spike artefacts were manually removed. The 20 channels were then inspected for their gain and coefficient of variation $(\mathrm{CV})$ scores which indicated the noise level in the signal. Channels with a gain $>8$ and $\mathrm{CV}>7.5$ were deemed to have significant noise and subsequently discarded. A band-pass filter of 0.01-0.2 $\mathrm{Hz}$ was applied on the remaining channels to eliminate baseline shift variations and slow signals. To compute hemoglobin levels for each channel, pre-processed signals were converted to changes in concentration of oxygenated $(\mathrm{HbO})$ and deoxygenated hemoglobin $(\mathrm{HbR})$ as a function of the modified Beer-Lambert law. The resulting haemodynamic signals were visually examined by two independent coders to inspect for any further artifacts. Any artifacts detected by the coders were removed.

The signal quality control eliminated 746 channels for the children who watched the videos with their father (out of 3114, 24.0\%) and 1290 for children who watched the videos with their mother (out of $3564,36.2 \%$ ). This is in line with other fNIRS studies.

\subsection{Analytical Plan}

fNIRS channels are interdependent: to favour a practical interpretation of the findings, we grouped the signals of fNIRS channels to examine the response of specific prefrontal areas. The channels were then divided into four groups based on their physical proximity to form the frontal left (channels 4, 6, 7, 11), frontal right (channels 13, 14, 16, 19), medial left (channels 1, 2, 3, 5, 8), and medial right (channels 10, 15, 17, $18,20)$ clusters. The brain activity signal of each cluster was derived by averaging the normalized signals of the channels composing each cluster. To ensure the quality of cluster signals, the cluster brain activity signal was computed for a participant if at least 3 channels with good quality signals were available.

Finally, we computed the average in the time window corresponding to the first 30 seconds of each video. This measure was used to quantify the PFC activity of each cluster and subject. A two-way ANOVA was conducted to evaluate the effect of type of parent, cluster, type of stimulus and gender of the child and their interaction on PFC activity. This approach allowed to consider the role of the whole PFC area, also accounting for localized effects, similar to (Fishburn et al., 2019; Moriarty, Bourbeau, Bellovary, \& Zuhl, 2019).

The interactions that led to significant effects were further investigated with posthoc tests. 

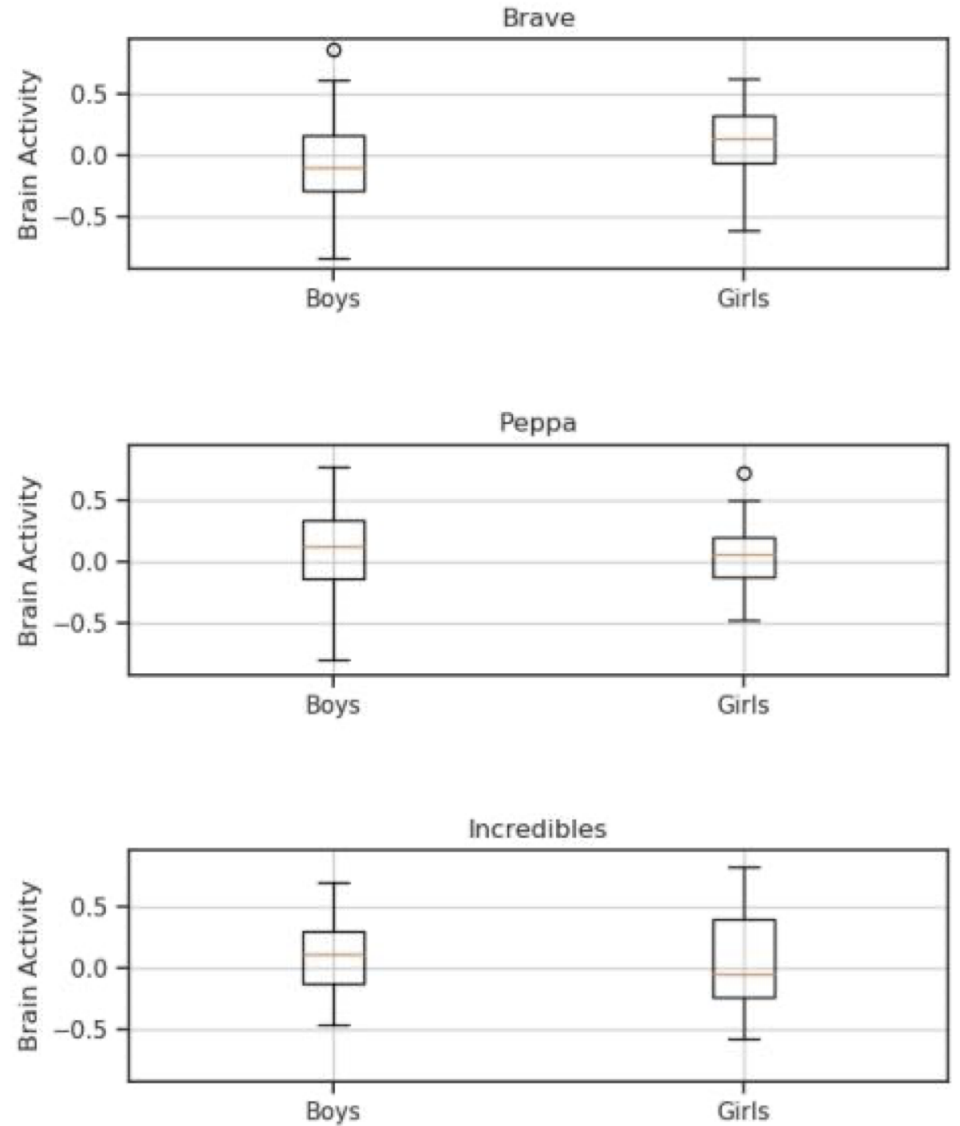

Figure 3. Brain activity of Boys and Girls for each type of stimulus.

\section{Results}

We found a significant main effect of type of parent on whole PFC activity $(\mathrm{F}(1,484)$ $=5.358, \mathrm{p}=0.021)$ indicating a significant different brain activity between children who watched the video with mothers $(\mathrm{M}=0.016, \mathrm{SD}=0.323)$ and with fathers $(\mathrm{M}$ $=0.077, \mathrm{SD}=0.309$ ).

The interaction effect of type of stimulus and gender of child was also significant $(\mathrm{F}(2,484)=6.086, \mathrm{p}=.002)$. A post-hoc Mann-Whitney U-test was applied to investigate the difference between Boys and Girls for each type of video. Significant differences were found for the Brave video $(\mathrm{U}=2564, \mathrm{p}=0.0003)$, no differences were found for Peppa $(\mathrm{U}=4207, \mathrm{p}=0.204)$ and Incredibles $(\mathrm{U}=4036, \mathrm{p}=0.325)$ (see Figure 3). 


\section{Discussion}

The present study aimed to investigate the difference in PFC activity in preschoolers while watching video clips with varying emotional valence (positive, negative, neutral). We formulated three hypotheses. One, we predicted stronger PFC activity in children while joint-viewing with their fathers than with their mothers. Two, we predicted stronger PFC activity in girls than in boys while watching positive, but not negative or neutral stimuli. Three, we predicted differences in PFC activity across varying emotional valences. We found support for the first two hypotheses. Although no significant main effects were found for emotional valence, we did find a significant interaction between gender difference and emotional valence.

The difference in PFC activity when joint-viewing with each parent suggests that the children may be processing the information in the videos differently. Our findings show stronger PFC activity of children in father-child than in mother-child dyads. Broadly, the presence of the parent may influence children's response to emotional stimuli (Coan, 2011). Previous studies have demonstrated systematic changes in physiological measures of children engaged in a task in the presence of a parent - mothers in most cases - compared to with a stranger or when alone (Gee et al., 2014; Keene et al., 2019; Myruski et al., 2019). However, there is a paucity in research involving fathers in such experimental designs. In this study, each child sat on the parent's lap throughout the screening. We can speculate that the PFC activity may reflect the way each parent engages their child during the task. Indeed, some studies have observed that mothers and fathers differ in their interactions with their child (Aznar \& Tenenbaum, 2016; Clarke-Stewart, 1978; Collins \& Russell, 1991; Lewis \& Lamb, 2003). Multiple possible mechanisms may underlie the influence of parental presence on PFC activity in children.

One possibility may be that mothers provide more external regulation for the child to process the emotional information in the videos (Coan, 2011; Gee et al., 2014). The lower PFC activity may indicate lower levels of self-regulation by the child (Ochsner \& Gross, 2005). In other words, the regulatory process is distributed between the child and parent, with less burden in children watching with their mothers. Another possibility may be that children exhibiting higher PFC activity are better able to mentalise with the characters in the video clips (Richardson \& Saxe, 2020). This suggests that the father's presence may better facilitate mentalisation during a joint attention task. Yet another mechanism, the PFC activity simply reflects the amount of mental workload exerted by the child while watching (Herff et al., 2014). Further research is required to isolate the influences of each parent's presence in the PFC activity of their children during such tasks.

The pattern in PFC activity also varied by the child's gender and video clips' emotional valence. Girls showed stronger PFC activity than boys but only when presented with positive stimuli. One review showed that preschoolers are likely to have a general positivity bias, where positive emotions are more efficiently processed than negative ones (Kauschke et al., 2019). This bias is likely due to the more sheltered, and hence more positive experiences among young children. One possible explanation as to why girls showed stronger PFC activity is due to their larger emotion-based knowledge (Barrett, Lane, Sechrest, \& Schwartz, 2000). With more accessible emotional experiences, even girls at a young age can demonstrate prowess in emotional regulation over boys, at least in response to positive stimuli. Alternatively, this gender difference may indicate a case of mirroring (Christov-Moore et al., 2014). The positive stimulus using a movie clip from Brave featured predominantly female main characters. In this case, 
the increased PFC activity in the girls may reflect their ability to better relate to the female lead than the boys. Future studies can measure how well the child identifies with the characters of the clips to disentangle these processes. Conversely, the similarities in PFC activity to neutral and negative stimuli may be due to either lack of experience with negative emotional experiences or similar degrees of mirroring with the characters in the clips.

The possibility that the presence of parents can have a moderating influence on children's ability to self-regulate when presented with stimuli of varying emotional valence can have long-term implications, particularly in later development (McClelland et al., 2018). Children at this age undergo an important stage of development in selfregulation (Kochanska, Murray, \& Harlan, 2000), as well as, self-awareness (Povinelli, Landry, Theall, Clark, \& Castille, 1999). The successful development of these social processes in early childhood predicts future socioemotional development and outcomes (Cosentino-Rocha \& Linhares, 2013). These outcomes can translate to later real-life consequences in areas, such as, academic achievement. For instance, von Suchodoletz and colleagues demonstrated that self-regulation is likely to be a fundamental skill for children to learn more effectively, particularly prior to and during formal school settings (Von Suchodoletz et al., 2013). Consequently, parents play an essential role in setting expectations for the child's experience with emotional stimuli (Hofer, 2006). Parents being present during such encounters during a child's early development sets a precedent for the child to determine the availability of cognitive resources to regulate positive and negative stimuli. Moreover, parents can instil more effective emotional regulation through active scaffolding (e.g. parents engaging in verbal reappraisal of negatively valenced emotional stimuli with their child), which improves the reappraisal of negative emotions much more than simply being present (Myruski et al., 2019). It is important to note that the effectiveness of parental presence may be short-lived and constrained by a critical period in early to middle childhood (Gee et al., 2014). These parental influences on children's early self-regulatory processing can therefore set a precedence for later development and outcomes. However, if mothers and fathers do indeed serve different functions, this calls to questions possible direct and indirect effects on their child's self-regulation skills (Lewis \& Lamb, 2003).

Notwithstanding these findings, we outline some of its limitations. First, the relatively small sample size may have reduced the power of groups and hence, underestimated the results. Larger samples may reveal more interaction effects and allow for cluster analysis to narrow down the specific prefrontal regions involved. Second, objective measures (e.g. eye gaze) during the viewing task can reveal behavioural patterns influenced by parental presence. Thirdly, future studies should include a control group with the child alone as a baseline for PFC activity. Fourthly, further research can examine the difference in dynamics of same-sex (e.g. father-son) and opposite-sex (e.g. father-daughter) dyads (Lovas, 2005). This adds another dimension of complexity to the difference in parental modulation of neural response to emotional stimuli. Finally, the mother-child and father-child dyads in this study were not from the same family. Analysing the PFC activity of children with their mother and father would provide a more accurate comparison of each parent's influence.

Overall, this is the first study to directly compare the influence of physical parental presence on neural activity of children in a joint attention task in mother-child and father-child dyads. We found that children showed stronger PFC activity when watching with their fathers than with their mothers. Moreover, girls show stronger PFC activity than boys when presented with positive stimuli. These findings emphasise the importance of understanding the parents' role and gender differences in neural 
responses during joint-viewing of video content.

\section{Acknowledgement(s)}

We would like to thank other research members of the Social and Affective Neuroscience lab for their assistance in this work.

\section{Disclosure statement}

The authors declare no conflict of interest. The funders had no role in the design of the study; in the collection, analyses, or interpretation of data; in the writing of the manuscript, or in the decision to publish the results.

\section{Funding}

This work was supported by the 2015 NAP Start-up Grant M4081597 (GE) from Nanyang Technological University Singapore and the Ministry of Education Tier-1 Grant (GE).

\section{Notes on contributor(s)}

Conceptualization, AA and GE; analysis, AB; investigation, JRD, JPMB, AA, ML, GG and GE; data curation, AA and AB; writing-original draft preparation, JRD, JPMB and AA; writing-review and editing, AA, ML, GG and GE; visualization, JRD, JPMB, AA and AB; funding acquisition GE.

\section{References}

Azhari, A., Leck, W., Gabrieli, G., Bizzego, A., Rigo, P., Setoh, P., ... Esposito, G. (2019). Parenting stress undermines mother-child brain-to-brain synchrony: A hyperscanning study. Scientific reports, 9(1), 1-9.

Azhari, A., Lim, M., Bizzego, A., Gabrieli, G., Bornstein, M. H., \& Esposito, G. (2020). Physical presence of spouse enhances brain-to-brain synchrony in co-parenting couples. Scientific reports, 10(1), 1-11.

Aznar, A., \& Tenenbaum, H. R. (2016). Parent-child positive touch: Gender, age, and task differences. Journal of nonverbal behavior, 40(4), 317-333.

Baptista, J., Osório, A., Martins, E. C., Castiajo, P., Barreto, A. L., Mateus, V., ... Martins, C. (2017). Maternal and paternal mental-state talk and executive function in preschool children. Social Development, 26(1), 129-145.

Barrett, L. F., Lane, R. D., Sechrest, L., \& Schwartz, G. E. (2000). Sex differences in emotional awareness. Personality and Social Psychology Bulletin, 26 (9), 1027-1035.

Blair, C. (2016). Developmental science and executive function. Current Directions in Psychological Science, 25(1), 3-7.

Bucy, E. P., Lang, A., Potter, R. F., \& Grabe, M. E. (1999). Formal features of cyberspace: Relationships between web page complexity and site traffic. Journal of the American Society for Information Science, 50(13), 1246-1256. 
Cabrera, N., Tamis-LeMonda, C. S., Bradley, R. H., Hofferth, S., \& Lamb, M. E. (2000). Fatherhood in the twenty-first century. Child development, 71(1), 127-136.

Carter, B., Rees, P., Hale, L., Bhattacharjee, D., \& Paradkar, M. S. (2016). Association between portable screen-based media device access or use and sleep outcomes: a systematic review and meta-analysis. JAMA pediatrics, 170(12), 1202-1208.

Charman, T., Ruffman, T., \& Clements, W. (2002). Is there a gender difference in false belief development? Social Development, 11(1), 1-10.

Cherland, E. (2004). The development of emotional competence. The Canadian child and adolescent psychiatry review, $13(4), 121$.

Christov-Moore, L., Simpson, E. A., Coudé, G., Grigaityte, K., Iacoboni, M., \& Ferrari, P. F. (2014). Empathy: gender effects in brain and behavior. Neuroscience \& Biobehavioral Reviews, 46, 604-627.

Clarke-Stewart, K. A. (1978). And daddy makes three: The father's impact on mother and young child. Child development, 466-478.

Coan, J. A. (2008). Toward a neuroscience of attachment. The Guilford Press.

Coan, J. A. (2011). The social regulation of emotion. In The oxford handbook of social neuroscience.

Collins, W. A., \& Russell, G. (1991). Mother-child and father-child relationships in middle childhood and adolescence: A developmental analysis. Developmental review, 11(2), 99136.

Cosentino-Rocha, L., \& Linhares, M. B. M. (2013). Temperamento de crianças e diferenças de gênero. Paidéia (Ribeirão Preto), 23(54), 63-72.

Denison, R. N., Driver, J., \& Ruff, C. C. (2013). Temporal structure and complexity affect audio-visual correspondence detection. Frontiers in Psychology, 3, 619.

Fishburn, F. A., Hlutkowsky, C. O., Bemis, L. M., Huppert, T. J., Wakschlag, L. S., \& Perlman, S. B. (2019). Irritability uniquely predicts prefrontal cortex activation during preschool inhibitory control among all temperament domains: A lasso approach. Neuroimage, 184, 68-77.

Frijda, N. H., et al. (1986). The emotions. Cambridge University Press.

Gamer, M., Lemon, J., Gamer, M. M., Robinson, A., \& Kendall's, W. (2012). Package 'irr'. Various coefficients of interrater reliability and agreement.

Gao, X., Maurer, D., \& Nishimura, M. (2010). Similarities and differences in the perceptual structure of facial expressions of children and adults. Journal of Experimental Child Psychology, 105(1-2), 98-115.

Gee, D. G., Gabard-Durnam, L., Telzer, E. H., Humphreys, K. L., Goff, B., Shapiro, M., ... others (2014). Maternal buffering of human amygdala-prefrontal circuitry during childhood but not during adolescence. Psychological science, 25 (11), 2067-2078.

Gogtay, N., Giedd, J. N., Lusk, L., Hayashi, K. M., Greenstein, D., Vaituzis, A. C., ... others (2004). Dynamic mapping of human cortical development during childhood through early adulthood. Proceedings of the National Academy of Sciences, 101(21), 8174-8179.

Hasson, U., \& Honey, C. J. (2012). Future trends in neuroimaging: Neural processes as expressed within real-life contexts. NeuroImage, 62(2), 1272-1278.

Herff, C., Heger, D., Fortmann, O., Hennrich, J., Putze, F., \& Schultz, T. (2014). Mental workload during n-back task - quantified in the prefrontal cortex using fnirs. Frontiers in human neuroscience, $7,935$.

Hofer, M. A. (2006). Psychobiological roots of early attachment. Current Directions in Psychological Science, 15(2), 84-88.

Kauschke, C., Bahn, D., Vesker, M., \& Schwarzer, G. (2019). The role of emotional valence for the processing of facial and verbal stimuli-positivity or negativity bias? Frontiers in psychology, 10, 1654.

Keene, J. R., Rasmussen, E. E., Berke, C. K., Densley, R. L., Loof, T., Adams, R. B., ... Marshall, A. (2019). The effect of plot explicit, educational explicit, and implicit inference information and coviewing on children's internal and external cognitive processing. Journal of Applied Communication Research, 47(2), 153-174. 
Kensinger, E. A., \& Schacter, D. L. (2006). Processing emotional pictures and words: Effects of valence and arousal. Cognitive, Affective, \& Behavioral Neuroscience, 6(2), 110-126.

Kiel, E. J., \& Kalomiris, A. E. (2015). Current themes in understanding children's emotion regulation as developing from within the parent-child relationship. Current opinion in psychology, 3, 11-16.

Kochanska, G., Murray, K. T., \& Harlan, E. T. (2000). Effortful control in early childhood: continuity and change, antecedents, and implications for social development. Developmental psychology, 36(2), 220.

Lepp anen, J. M., \& Hietanen, J. K. (2004). Positive facial expressions are recognized faster than negative facial expressions, but why? Psychological research, 69(1-2), 22-29.

Lewis, C., \& Lamb, M. E. (2003). Fathers' influences on children's development: The evidence from two-parent families. European journal of psychology of education, 18(2), 211-228.

Lovas, G. S. (2005). Gender and patterns of emotional availability in mother-toddler and father-toddler dyads. Infant Mental Health Journal: Official Publication of The World Association for Infant Mental Health, 26(4), 327-353.

Luciana, M., \& Nelson, C. A. (1998). The functional emergence of prefrontally-guided working memory systems in four-to eight-year-old children. Neuropsychologia, 36(3), 273-293.

McClelland, M., Geldhof, J., Morrison, F., Gestsdóttir, S., Cameron, C., Bowers, E., ... Grammer, J. (2018). Self-regulation. In N. Halfon, C. B. Forrest, R. M. Lerner, \& E. M. Faustman (Eds.), Handbook of life course health development (pp. 275-298). Springer International Publishing.

McClure, E. B. (2000). A meta-analytic review of sex differences in facial expression processing and their development in infants, children, and adolescents. Psychological bulletin, 126(3), 424.

Meuwissen, A. (2017). How do parents affect preschoolers' self-regulation? establishing the role of autonomy supportive parenting.

Moriarty, T., Bourbeau, K., Bellovary, B., \& Zuhl, M. N. (2019). Exercise intensity influences prefrontal cortex oxygenation during cognitive testing. Behavioral Sciences, 9(8), 83.

Moriguchi, Y., \& Hiraki, K. (2009). Neural origin of cognitive shifting in young children. Proceedings of the National Academy of Sciences, 106 (14), 6017-6021.

Myruski, S., Birk, S., Karasawa, M., Kamikubo, A., Kazama, M., Hirabayashi, H., \& DennisTiwary, T. (2019). Neural signatures of child cognitive emotion regulation are bolstered by parental social regulation in two cultures. Social cognitive and affective neuroscience, $14(9), 947-956$.

Ochsner, K. N., \& Gross, J. J. (2005). The cognitive control of emotion. Trends in cognitive sciences, 9(5), 242-249.

Povinelli, D. J., Landry, A. M., Theall, L. A., Clark, B. R., \& Castille, C. M. (1999). Development of young children's understanding that the recent past is causally bound to the present. Developmental Psychology, 35(6), 1426.

Premack, D., \& Woodruff, G. (1978). Does the chimpanzee have a theory of mind? Behavioral and brain sciences, $1(4), 515-526$.

Reeck, C., Ames, D. R., \& Ochsner, K. N. (2016). The social regulation of emotion: An integrative, cross-disciplinary model. Trends in cognitive sciences, 20(1), 47-63.

Richardson, H., Lisandrelli, G., Riobueno-Naylor, A., \& Saxe, R. (2018). Development of the social brain from age three to twelve years. Nature communications, 9(1), 1-12.

Richardson, H., \& Saxe, R. (2020). Development of predictive responses in theory of mind brain regions. Developmental science, 23(1), e12863.

Saxe, R., \& Kanwisher, N. (2003). People thinking about thinking people: the role of the temporo-parietal junction in "theory of mind". Neuroimage, 19(4), 1835-1842.

Schaefer, A., Nils, F., Sanchez, X., \& Philippot, P. (2010). Assessing the effectiveness of a large database of emotion-eliciting films: A new tool for emotion researchers. Cognition and emotion, 24(7), 1153-1172.

Von Suchodoletz, A., Gestsdottir, S., Wanless, S. B., McClelland, M. M., Birgisdottir, F., Gunzenhauser, C., \& Ragnarsdottir, H. (2013). Behavioral self-regulation and relations to 
emergent academic skills among children in germany and iceland. Early Childhood Research Quarterly, 28(1), 62-73.

Walker, S. (2005). Gender differences in the relationship between young children's peerrelated social competence and individual differences in theory of mind. The Journal of genetic psychology, 166(3), 297-312.

Yeshurun, Y., Nguyen, M., \& Hasson, U. (2017). Amplification of local changes along the timescale processing hierarchy. Proceedings of the National Academy of Sciences, 114(35), 9475-9480. 